\title{
CORRIGENDUM
}

\section{Experimental study on water-wave trapped modes - CORRIGENDUM}

\author{
P. J. Cobelli, V. Pagneux, A. Maurel and P. Petitjeans \\ doi:10.1017/S0022112010004222, Published by Cambridge University Press, \\ 6 January 2011
}

In the article published by Cobelli et al. (2011), the authors forgot to make reference to their original work published in Cobelli et al. (2009). They also did not cite the source of figures 1, 6, 12 and 21, first published in Cobelli et al. (2009). Retrospective permission to use the figures have been granted by the Editor of EPL.

The second paragraph on p. 449 should read:

The existence of trapped modes around a cylinder in a waveguide was identified by Cobelli et al. (2009) where no parametric dependences were explored. The aim of this study is to gain further understanding regarding the occurrence of trapped modes of both NS and NA types, the dependence of their eigenfrequencies with geometry, and the characterization of their detailed spatial structure.

\section{REFERENCES}

Cobelli, P., Pagneux, V., Maurel, A. \& Petitjeans, P. 2009 Experimental observation of trapped modes in a water wave channel. Europhys. Lett. 88, 20006. doi:10.1209/0295-5075/88/20006.

Cobelli, P., Pagneux, V., Maurel, A. \& Petitjeans, P. 2011 Experimental study on water-wave trapped modes. J. Fluid Mech. 666, 445-476. doi:10.1017/S0022112010004222. 